\title{
Electric Flora
}

\author{
Harnessing electrostatic energy \\ from the body to power a forest of light.
}

\section{Describe what you made.}

Electric Flora is an

installation that creates a space of interactivity by harvesting electrostatic energy from the human body. Our idea is to create a jungle of interactive plants that are suspended from the ceiling. When the user touches the conductive tip of the plants, made from acrylic tubes with an array of LEDs, the leaves light up. The only power source of the installation is the user's own energy/electrical charge. The user wears Teflon shoes to amplify the body's electrostatic charge.

\section{What for you is the most important/interesting thing about what you made?} The "technology" we are using in this project is based on a type of contact electrification called the tribo-electric effect. Everyone has experienced this phenomenon; in dry winter air we easily charge ourselves up and get little shocks by touching metal surfaces. The principle of this electrification is very simple, but at the same time it's fascinating to explore our body as a cloud of atoms and electrons and as a carrier of electrical charge. In this installation, you can even feel how the charge is floating through your body when you are touching the conductive end of the acrylic tubes. Besides offering the body experience in space, the

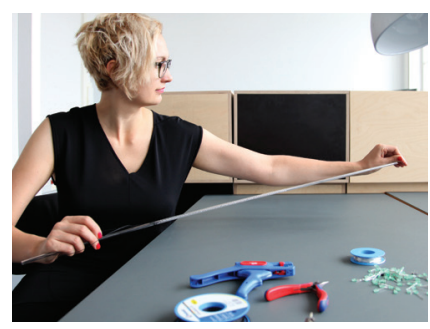

$\rightarrow$ Embedding wires in the acrylic tube.

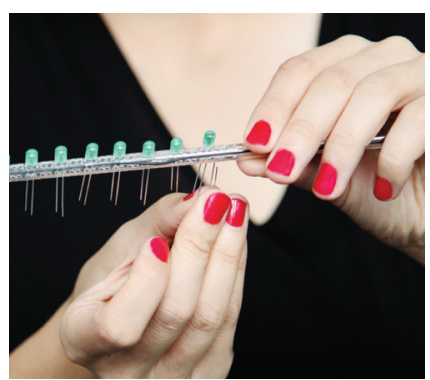

$\rightarrow$ Fitting the LEDs through the holes.

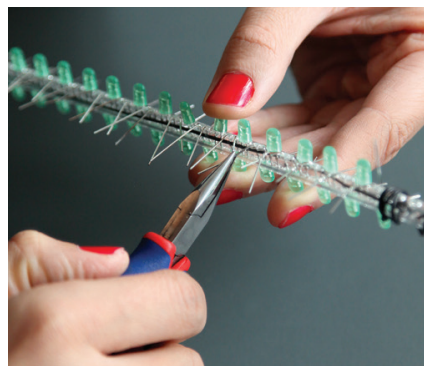

$\rightarrow$ Connecting the LEDs.

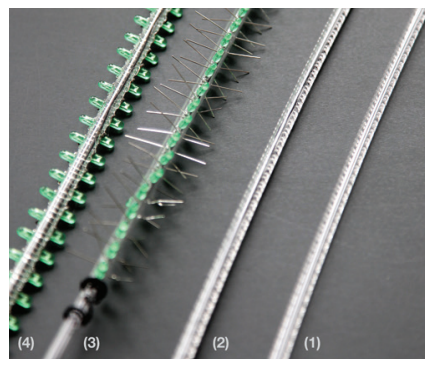

$\rightarrow$ Four stages of the acrylic rod: (1) the laser cut acrylic rod with holes (2) the rod with the wires embedded (3) the rod with the LEDs embedded (4) the finished rod with LEDs connected.

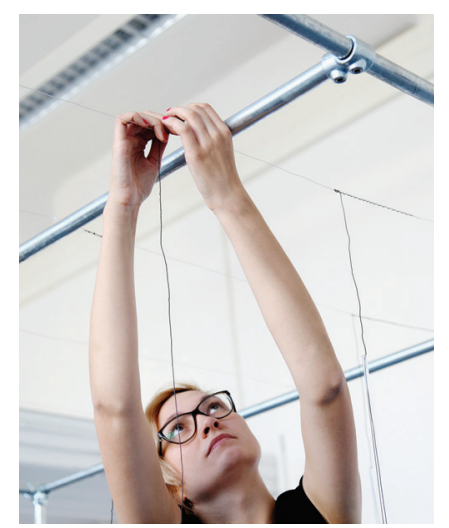

$\rightarrow$ Hanging the rods to the metal structure.

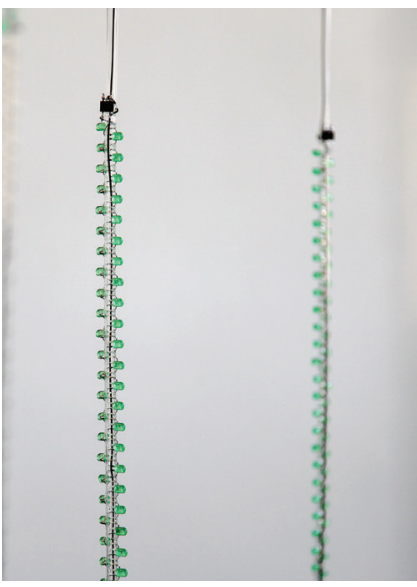

$\rightarrow$ The wires connected to the LEDs series and the rectifier (converting $\mathrm{AC}$ to $\mathrm{DC}$ ).

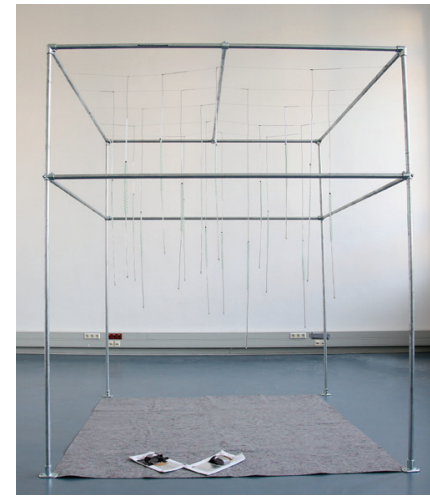

$\rightarrow$ The whole installation setup, including carpet and Teflon shoes. project opens the discussion about using electrostatic energy as a source of power in the future.

\section{Was this a collaborative process, and if so, who was involved? Electric} Flora is an outcome of a collaboration between Disney Research Pittsburgh and the Berlin University of Arts. After exploring the use of electrostatic energy for interactive books (in the project Paper Generators, Disney Research Pittsburgh) we wanted to take advantage of the low-cost technology of harvesting electrostatic energy and use it in a big installation in space where the whole body is generating energy.

\section{Did anything go wrong?}

We totally underestimated the influence of atmospheric and environmental conditions. We were developing and testing the system in our lab, where the temperature is constant and the humidity is always around 10 percent because of the air conditioning and climate control. When we set up the installation for the first time in a gallery space, we realized the big impact of the weather on the output. On a warmer rainy day you had to do a vigorous workout with the Teflon shoes to get a bright light. However if it was cold and dry outside, it was even 
